\title{
p120-catenin is essential for maintenance of barrier function and intestinal homeostasis in mice
}

\author{
Whitney G. Smalley-Freed, ${ }^{1}$ Andrey Efimov, ${ }^{1}$ Patrick E. Burnett, ${ }^{1,2,3}$ \\ Sarah P. Short, ${ }^{1}$ Michael A. Davis, ${ }^{4}$ Deborah L. Gumucio, ${ }^{5}$ \\ M. Kay Washington, ${ }^{6}$ Robert J. Coffey, ${ }^{3,7,8}$ and Albert B. Reynolds ${ }^{1}$
}

\begin{abstract}
'Department of Cancer Biology, Vanderbilt University, Nashville, Tennessee, USA. ${ }^{2}$ Department of Medicine, Division of Dermatology, Vanderbilt University School of Medicine, Nashville, Tennessee, USA. ${ }^{3}$ VA Tennessee Valley Healthcare System, Medical Service, Nashville, Tennessee, USA. ${ }^{4}$ Department of Human Biology, Fred Hutchinson Cancer Research Center, Seattle, Washington, USA. ${ }^{5}$ Department of Cell and Developmental Biology, University of Michigan Medical School, Ann Arbor, Michigan, USA.

${ }^{6}$ Department of Pathology, Vanderbilt University Medical Center, Nashville, Tennessee, USA. ${ }^{7}$ Cell and Developmental Biology, Vanderbilt University, Nashville, Tennessee, USA. ${ }^{8}$ Department of Medicine, Vanderbilt University Medical School, Nashville, Tennessee, USA.
\end{abstract}

\begin{abstract}
Epithelial-cadherin (E-cadherin) is a master organizer of the epithelial phenotype. Its function is regulated in part by $\mathrm{p} 120$-catenin (referred to herein as $\mathrm{p} 120$ ), a cytoplasmic binding partner that directly regulates cadherin stability. As it has been suggested that cadherins have a role in inflammatory bowel disease (IBD), we sought to investigate this further by assessing the effect of $\mathrm{p} 120$ deficiency in mouse small intestine and colon. p120 conditional KO mice were superficially normal at birth but declined rapidly and died within 21 days. Cellcell adhesion defects and inflammation led to progressive mucosal erosion and terminal bleeding, similar to what is observed in a dominant-negative cadherin mouse model of IBD. Additionally, selective loss of adherens junctions and accumulation of atypical COX-2-expressing neutrophils in $\mathrm{p} 120$-null areas of the colon were observed. To elucidate the mechanism, direct effects of $\mathrm{p} 120$ deficiency were assessed in vitro in a polarizing colon cancer cell line. Notably, transepithelial electrical resistance was dramatically reduced, neutrophil binding was increased 30 fold, and levels of COX-2, an enzyme associated with IBD, were markedly increased in neutrophils. Our data suggest that p120 loss disrupts the neonatal intestinal barrier and amplifies neutrophil engagement and that these changes lead to catastrophic inflammation during colonization of the neonatal gut with bacteria and other luminal antigens. Thus, we conclude that $\mathrm{p} 120$ has an essential role in barrier function and epithelial homeostasis and survival in the intestine.
\end{abstract}

\section{Introduction}

Classical cadherins comprise a family of transmembrane cell-cell adhesion receptors important in development, morphogenesis, and cancer (reviewed in refs. 1 and 2). The main cadherin in epithelial cells, epithelial-cadherin (E-cadherin), is widely regarded as a master organizer of the epithelial phenotype (2). E-cadherin mutation is causally associated with familial gastric cancer (3) and lobular carcinoma of the breast $(4,5)$, in which it functions as a classic tumor suppressor. However, it is better known as a metastasis suppressor, because its frequent downregulation in advanced tumors plays a causal role in the transition to metastasis $(4,6)$. These observations underpin wide interest in E-cadherin and in the catenins, cytoplasmic binding partners that regulate cadherin function.

p120-catenin (hereafter referred to as p120) and $\beta$-catenin are armadillo repeat proteins that interact directly with distinct sites on the cadherin tail. $\beta$-catenin interacts further with $\alpha$-catenin to modulate functional interactions with the actin cytoskeleton (7-9). In contrast, p120 appears to modulate the strength of cell-cell adhesion by controlling the stability and retention of E-cadherin at the cell surface $(10,11)$. p120 also modulates the activities of several Rho GTPases (12-15), suggesting that the catenins in general coordinate a functional interface between cadherins and the actin cytoskeleton.

Conflict of interest: The authors have declared that no conflict of interest exists. Citation for this article: J Clin Invest. 2010;120(6):1824-1835. doi:10.1172/JCI41414.
Other roles for $\mathrm{p} 120$ are suggested by its physical interaction with the transcription factor Kaiso $(16,17)$, which belongs to the POZ family of proteins, most of which have been implicated in development and cancer (18). Several lines of evidence suggest roles for p120 and Kaiso in canonical and noncanonical Wnt signaling (19-23).

Importantly, the stability of most, if not all, classical and type 2 cadherins is dependent on interaction with p120 $(10,11)$. When multiple cadherins are present in a cell, as is often the case, silencing p120 destabilizes all of them. Conversely, silencing a single cadherin leaves more p120 for the others, and their levels increase. This so called "p120 sharing" phenomenon highlights the importance of overall p120 levels as a cellular set point for determining overall levels of classical cadherins. It also explains, at least in part, why the effects of p120 ablation on cell-cell adhesion are often more profound than silencing individual cadherins.

Previous evidence suggests a role for cadherins in inflammatory bowel disease (IBD) and, by inference, a role for $\mathrm{p} 120$. In particular, transgenic expression of a dominant-negative cadherin (DN-cadherin) in the mouse small intestine induces a Crohn-like IBD by 3 months of age, and the majority of these animals develop adenomas within 6 months (24). IBDs (e.g., Crohn disease, ulcerative colitis) are chronically remitting inflammatory conditions, affecting over 1.4 million Americans (25). The etiology is poorly understood and probably multifactorial. Although many genetic markers have been identified $(26,27)$, none by themselves account for more than a fraction 

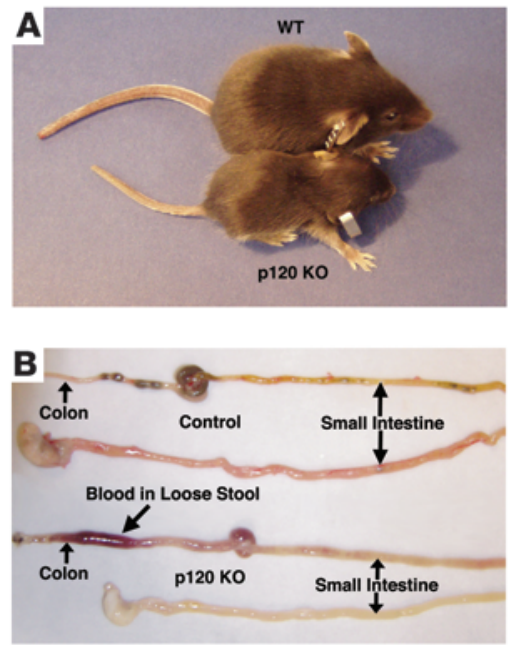

C

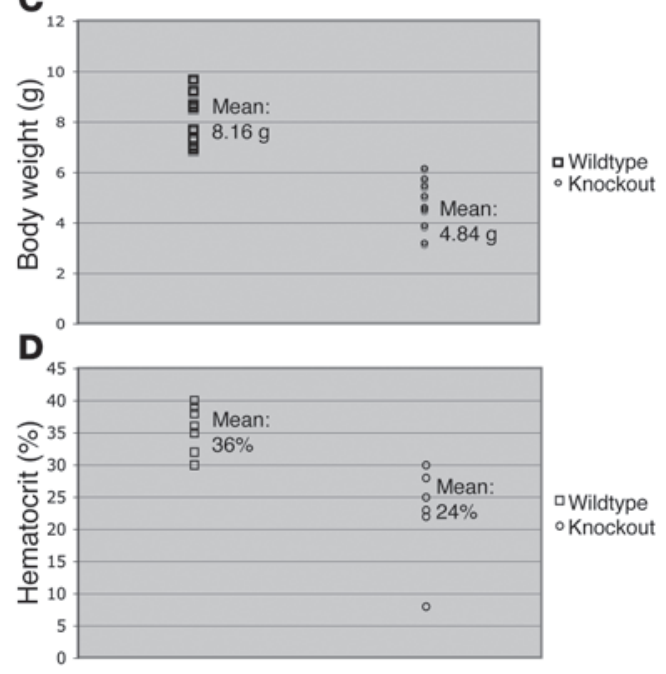

\section{Figure 1}

Clinical phenotype and cause of death. (A) Comparison of p120 KO and littermate control mice at 16 days. $\mathrm{KO}$ animals displayed an obvious failure to thrive. (B) Comparison of the GI tracts from control (top) and p120 KO (bottom) mice. The KO small intestine is pale white due to loss of blood. Note blood in the distended colon. (C) The p120 $\mathrm{KO}$ mice weigh on average about half as much as controls. Individual symbols represent individual mice. $P<0.0001$. (D) Hematocrits of p120 KO mice indicate severe anemia. $P=0.0007$. of IBD cases. In general, irrespective of genetic events, IBD is thought to result from an excessive immune response to luminal antigens. Several studies point to excess epithelial permeability as a primary etiologic factor $(28,29)$. In this scenario, an inappropriate immune response (and inflammation) is triggered by antigen leakage across a defective epithelial barrier. Conversely, inflammation itself can drive excess epithelial permeability $(30,31)$, making it difficult a priori to distinguish cause and effect. To this end, mouse models of IBD have been invaluable, because the initial event can be clearly defined. In general, the findings in mice have linked primary etiologies to genetic defects in (a) epithelial integrity/permeability, (b) innate immunity, and/or (c) adaptive immunity. It is now known that both immune and barrier defects can promote IBD, but the actual etiology in most IBD patients remains unclear and is probably influenced by a number of additional factors, including environmental context.

To directly study roles for p120 in vivo, conditional p120 KO mouse models have been generated by our group (32) and by the Reichardt laboratory (33). p120 KO in the intestine has not been described. However, the effects of p120 KO in other organ systems have been surprisingly tissue specific and largely unpredictable. For example, mammary gland development fails altogether in the absence of p120, whereas little or no effect is observed in the prostate (A.B. Reynolds, unpublished observations). Interestingly, p120 KO in the epithelium of the developing salivary gland completely blocks formation of acini, resulting in a gland composed entirely of grossly disfigured ducts. Cell-cell adhesion is markedly impaired in these structures, resulting in obvious disruption to the epithelial barrier (32). In striking contrast, p120 ablation in the epidermis has no discernible effect on either cell-cell adhesion or barrier function, despite similarly reduced levels of both E- and P-cadherins. Instead, these mice develop severe epidermal inflammation due to cell autonomous activation of NF-кB (34). Notably, the salivary gland (barrier defects) and epidermal (inflammation) phenotypes are major factors in the etiology of IBD.

Here, we report that p120 is essential in the intestine for epithelial homeostasis and life. p120 KO in the epithelium of the small and large intestines resulted in inflammation and mucosal damage, leading to bleeding and death within the first 3 weeks of life. In vitro and in vivo evidence show that $\mathrm{p} 120$ is essential for maintenance of barrier function. The barrier defect precedes the inflammatory response, which is probably exacerbated by accumulation of bacteria after birth. In addition, there appears to be a separate cell autonomous consequence of $\mathrm{p} 120$ downregulation that affects neutrophil behavior specifically, dramatically increased neutrophil attachment to p120deficient epithelial monolayers and selective upregulation of COX-2. These observations are consistent with a role for p120 in barrier maintenance, inflammation, and potentially IBD.

\section{Results}

p120 expression in the intestine is essential for life. Cre/Lox technology was used to selectively target p120 KO to the small intestine and colon. Our conditional p120 KO mouse has been used previously to target p120 ablation to the salivary gland (32) and skin (34). Here, we crossed floxed p120 mice ( $120^{\mathrm{F} / \mathrm{F}}$ mice) to a well-characterized villin-Cre mouse model (villin-Cre) engineered to selectively express Cre in the epithelium of the intestine (35). Villin is an actin bundling protein found in all enterocytes. Normally, it is also expressed in the kidney, but the model used here has selectively lost that function. Villin expression in the intestine increases as cells migrate from the crypts to the tips of villi (36). During embryonic development, Villin (Vill) gene expression reaches significant levels by $14-15$ days post coitum, at which time the intestinal epithelium undergoes extensive remodeling, including formation of villi (35). In the villin-Cre mice, cre activity is first detectable in the embryonic midgut and hindgut at 12.5 days post coitum (35).

p120 KO mice (villin-Cre/p120 F/F mice) were born in normal Mendelian ratios and indistinguishable from littermates. Signs of discomfort were not obvious until after day 10, at which point the $\mathrm{KO}$ animals declined rapidly. Virtually all p120 KO mice died between 11 and 23 days (average $=17$ days) after birth. By the time of death, p120 KO mice were significantly smaller than littermates $(n=4.84 \mathrm{~g}$ vs. $8.16 \mathrm{~g}$, respectively; $P<0.0001)$ (Figure 1, A and C), and all suffered from liquid bloody stools (Figure 1B).

By 12-14 days, KO mice were sluggish, with ruffled coats and hunched posture, consistent with dehydration. As shown in Figure 1A, WT and $\mathrm{KO}$ animals were photographed on day 16, just prior to the removal of their gastrointestinal (GI) tracts for gross examination (Figure $1 \mathrm{~B}$ ). Note that the $\mathrm{KO}$ tract is extremely pale relative to the WT tract, due to anemia. Indeed, severe loss of blood was the primary cause of death, as the hematocrits of p120 KO mice at day 16 
A

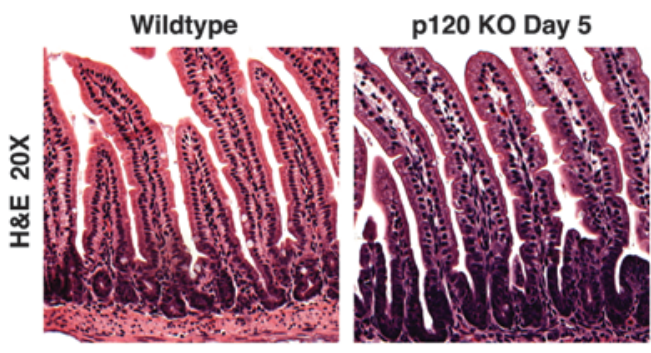

B
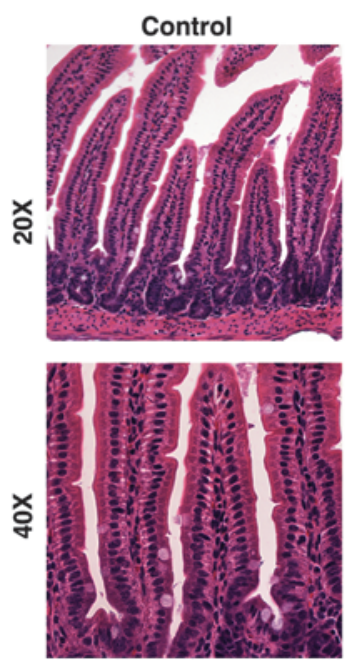

stine

p120 KO
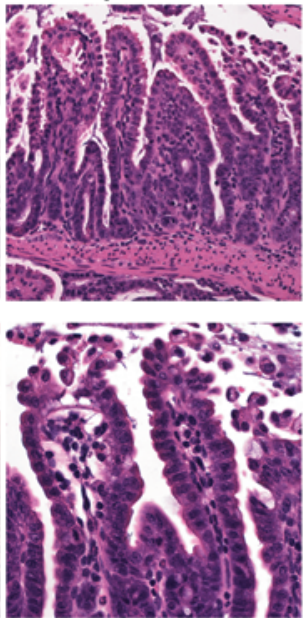

p120 KO Day 10
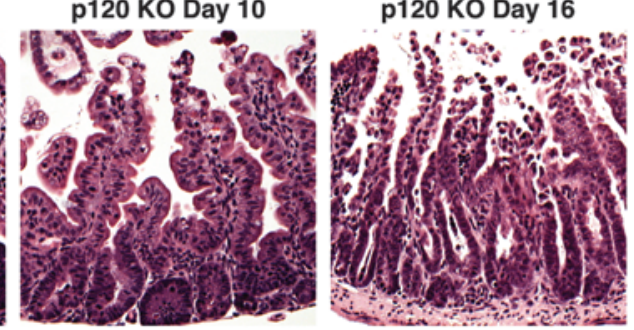

C

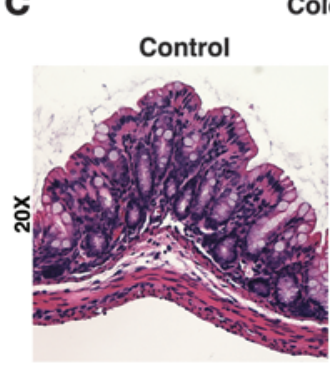

Colon
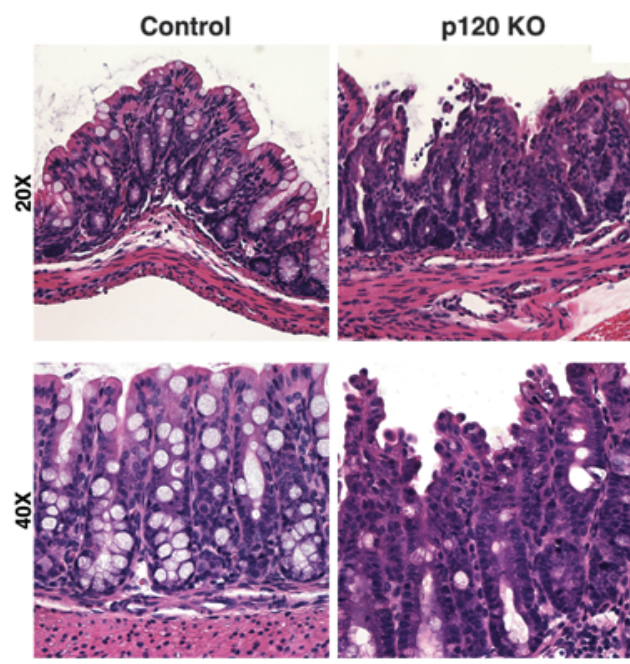

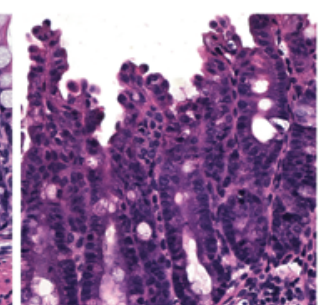

\section{Figure 2}

Rapid deterioration of gut morphology. Sections of small intestine or colon from $\mathrm{p} 120 \mathrm{KO}$ or control mice were analyzed by H\&E staining. (A) Sections of small intestine from day 5 , 10 , and 16 p120 KO mice. Note the rapid deterioration in villus morphology and cell-cell adhesion. Original magnification, $\times 20$. (B) Sections of control and $\mathrm{p} 120 \mathrm{KO}$ small intestines at day 16 . Note the morphologic defects, compromised cell-cell adhesion, and erosion of cells from the tips of villi (bottom right panel), which led to bleeding. (C) Sections of control and $\mathrm{p} 120 \mathrm{KO}$ colons at day 16. p120 KO causes a doubling of mucosal height (compare the images at a magnification of $\times 40$ ) and mucosal erosion (top right panel). Original magnification, $\times 20$ (B and $\mathbf{C}$, top rows); $\times 40$ (B and $\mathrm{C}$, bottom rows). were approaching levels incompatible with life (mean $=24 \%$; Figure 1D). All of the KO mice were similarly affected, indicating an essential role for $\mathrm{p} 120$ in the intestine.

p120 is required for maintenance of epithelial morphology and function. To evaluate effects of p120 ablation at the tissue level, samples from the intestine and colon were obtained at 5, 10, and 16 days after birth and embedded in paraffin for $\mathrm{H} \& \mathrm{E}$ staining (Figure 2). Minor differences between WT and p120 KO samples in the small intestine were apparent by day 5 and manifested primarily as slight rounding of some enterocytes along the villi (Figure 2A). By day 10, there were obvious abnormalities in villus and cell morphology (Figure 2A). At day 16, major defects in cell-cell adhesion were visible in both the small intestine and colon (Figure 2, B and C). Large numbers of poorly adhesive enterocytes prematurely blebbed off into the lumen (Figure 2B, right panels; Figure 2C, right panels). In the small intestine, the tops of villi were frequently blunted or broken off altogether, along with clusters of shed cells (e.g., Figure 2B, bottom right panel). Similarly, hobnail cells at the tops of crypts in the colonic epithelium (Figure 2C, right panels) were often sloughed off, leading to superficial erosion. These areas of severely compromised epithelium in the small and large intestine were frequently associated with free red blood cells, the only discernible source of the intestinal bleeding.

To visualize these effects at the ultrastructural level (Figure 3), we initially examined day 16 colonic tissue by EM at magnifications of $\times 2,650$ and $\times 15,000$ (Figure 3A). Significant gaps (Figure 3A, arrows) were observed along the entire basolateral surface in p 120 KO samples (compare control and p120 KO panels at magnifications of $\times 2,650)$. At higher levels of magnification $(\times 15,000)$, tight junctions were observed to be largely intact (Figure $3 \mathrm{~A}$, arrowhead), consistent with several other lines of evidence (i.e., Figure 4), showing that $\mathrm{p} 120 \mathrm{KO}$ selectively targets the adherens junctions. To determine whether the basolateral gaps were associated with inflammation, we repeated these experiments with colonic tissue from newborn WT and p120 KO mice (Figure 3B, compare control and p120 KO panels). These images indicate that the gaps observed at day 16 are also evident at birth, long before the detectable onset of inflammation.

To further characterize the KO, we double-immunostained paraffin-embedded sections with antibodies to p120 and E-cadherin (Figure 4). Interestingly, despite the severity of the phenotype, p120 $\mathrm{KO}$ throughout the intestine was mosaic, occurring in discreet patches involving no more than half of the total epithelial compartment. As illustrated by Figure 4, A and B, this mosaic pattern of p120 KO was characterized by alternating patches of p120-positive and -negative staining. Inefficient p120 excision is characteristic of this p120 floxed mouse model; approximately half of the epithelial organs targeted to date have, in fact, been mosaic, probably because the loxP sites are more than $8 \mathrm{~kb}$ apart. E-cadherin levels were selectively decreased in p120-null cells (Figure 4A, arrowhead), as compared with cells that retained p120 expression (Figure 4A, arrow). As expected, $\beta$-catenin and $\alpha$-catenin levels decreased in parallel with those of E-cadherin (Figure 4B). No cytoplasmic or nuclear $\beta$-catenin staining was observed in p120-null cells. In contrast to $\alpha$-and $\beta$-catenin, staining for desmoglein and occludin was largely unaffected by p120 loss (Figure 4, B and C, arrowheads), consistent with the presence of apparently normal desmosomes and tight junctions, respectively, as also observed in the previously described electron micrographs. Together, these data indicate that E-cadherin and catenin levels in the intestine are largely dependant on p120, whereas tight junctions and desmosomes are not. 
A Control

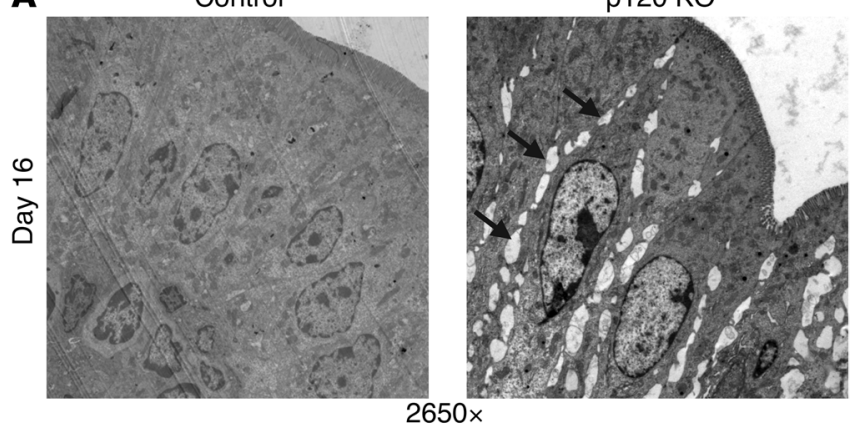

$2650 \times$

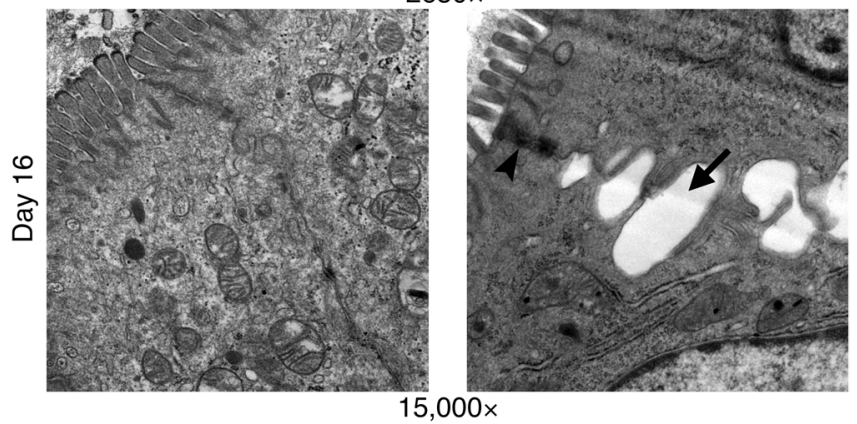

B

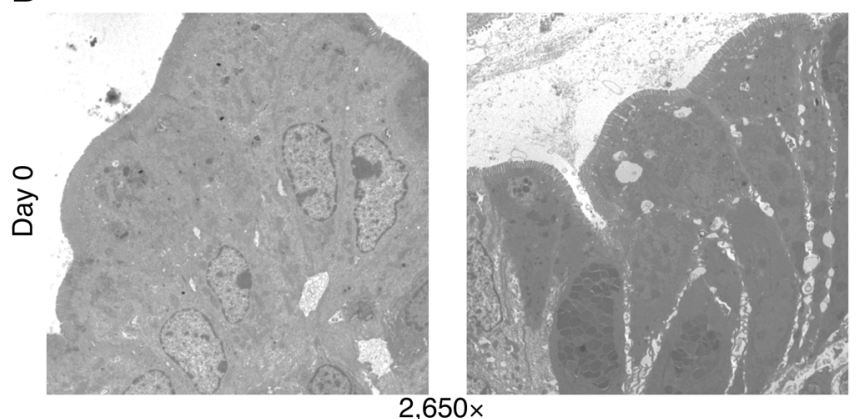

Despite major defects in cell morphology and adhesion, overall cell polarity in the $\mathrm{p} 120 \mathrm{KO}$ intestine was largely unaffected (Figure 5A). In the developing salivary gland, p120 ablation causes severe defects in cell polarity, as evidenced by mislocalization of the polarity markers Crumbs3 (apical) and $\mathrm{Na} / \mathrm{K}$ ATPase (basolateral) (32). In contrast, p120-null areas in the intestine maintained continuous Crumbs3 staining (Figure 5A), up until relatively late stages of mucosal deterioration. Likewise, localization of the basement membrane component laminin was not significantly altered by 120 ablation (Figure 5B). The basolateral marker Na/K ATPase localized correctly in spite of defects in adherens junctions and cell-cell adhesion (Figure 5A), as was also the case for F-actin (data not shown). These observations imply that p120 ablation by itself does not markedly effect cell polarity in the intestine.

Similarity to human IBDs. Although none of the p120 KO animals survived beyond 23 days, $\mathrm{H} \& \mathrm{E}$ analysis of colon tissue at day 16 revealed significant crypt hyperplasia and a doubling of mucosal height (e.g., Figure 2C, compare bottom panels), suggesting increased proliferation, decreased apoptosis, or both. To quantify enterocyte proliferation in vivo, BrdU was injected into the peritoneal cavity 2 hours prior to sacrifice, and BrdU incorporation was assayed by immunofluorescence (Figure 6A, compare left panels). Quantification of BrdU-positive nuclei revealed a 3-fold increase in proliferation

\section{Figure 3}

Characterization of cell-cell adhesion defects by EM. (A) At low magnification (original magnification, $\times 2,650$ ), the extent of the damage (arrows) is clearly visible along entire basolateral surfaces of the p120 KO cells. Electron micrographs of control and $\mathrm{p} 120 \mathrm{KO}$ colon are shown at higher magnification (original magnification, $\times 15,000$ ) in the bottom panels. Tight junctions remain intact (arrowhead), but adherens junctions are apparently replaced by significant gaps (arrow) along the length of the basolateral membrane. (B) Intercellular gaps are also visible in the p120 KO colon immediately after birth (day 0 ). Original magnification, $\times 2,650$.

of the $\mathrm{KO}$ colonic epithelium (mean $=9,653.5$ positive nuclei/colon) relative to the control counterpart (3,505.5 positive nuclei/colon) $(P<0.05)$ (data not shown) and a 2-fold increase in proliferation of the $\mathrm{KO}$ small intestine epithelium (mean $=30,336$ positive nuclei/ small intestine) relative to its control counterpart (14,531.5 positive nuclei/small intestine) $(P=0.01)$ (Figure 6A, compare control and p120 KO panels, left side). The bottom left panel of Figure $6 \mathrm{~A}$ shows a particularly good example of the mosaic pattern of p120 ablation in these mice. Although decreased apoptosis could potentially contribute to the thickening of the p120 KO mucosa, analysis of WT and KO samples by TUNEL and cleaved-caspase-3 assays did not reveal statistically significant differences (data not shown).

In the normal intestine, stem cells in the crypts give rise to new cells that differentiate into enterocytes, migrate upward over 3-5 days, and then slough off into the lumen. To examine the effect of p120 loss on cell migration, BrdU was injected 30 hours prior to sacrifice, and BrdU incorporation was assayed as above in WT and $\mathrm{KO}$ animals (Figure 6A, compare control and p120 KO panels, right side). Interestingly, cell migration was accelerated by 2 fold in the p120 KO animals (labelled cells migrate $60 \%$ of the way to the top of the villous in $\mathrm{KO}$ vs. $30 \%$ in WT; $P=0.0002)$. This experiment illustrates precocious arrival of labeled cells at the tops of villi in p120 KO animals. In the same time interval, labeled cells in control animals traveled less than half the distance.

In contrast to the pattern of E-cadherin suppression, which was clearly a cell autonomous consequence of p120 ablation, most other effects (e.g., proliferation and migration) were widespread and apparently unrelated to this pattern. This phenomenon is reminiscent of field effects generated by inflammation in IBD patients (37). To further examine this effect, we localized Ezrin, phosphoERM (pERM) (Figure 6B), and phospho-c-Jun (pJun) (Figure 6C), the former being a potential marker for Rho GTPase activity (38), the latter a marker of various forms of cellular stress, including inflammation (39). Comparison of the staining in WT and KO samples revealed differences consistent with inflammation, but in contrast to E-cadherin staining, these markers did not mirror the mosaic pattern of 120 staining present throughout KO tissue.

Field effects in IBD reflect widespread distribution of inflammatory cytokines and the presence of various types of immune cells (37). H\&E staining of tissue from 16-day-old p120 KO mice revealed an increase in inflammatory cells in the lamina propria of the colon but not the small intestine. Paraffin-embedded sections from WT and p120 KO mice were therefore stained with markers for neutrophils, macrophages, and lymphocytes (Figure 7). Widespread infiltration of neutrophils was observed in the colons of p120 KO mice but not in the small intestines or in control tissue (Figure 7A, compare control and p120 KO panels, left side). Macrophage numbers, on the other hand, were similar in WT and control sections (Figure 7A, middle panels). The $\mathrm{CD}^{+} \mathrm{T}$ cell num- 

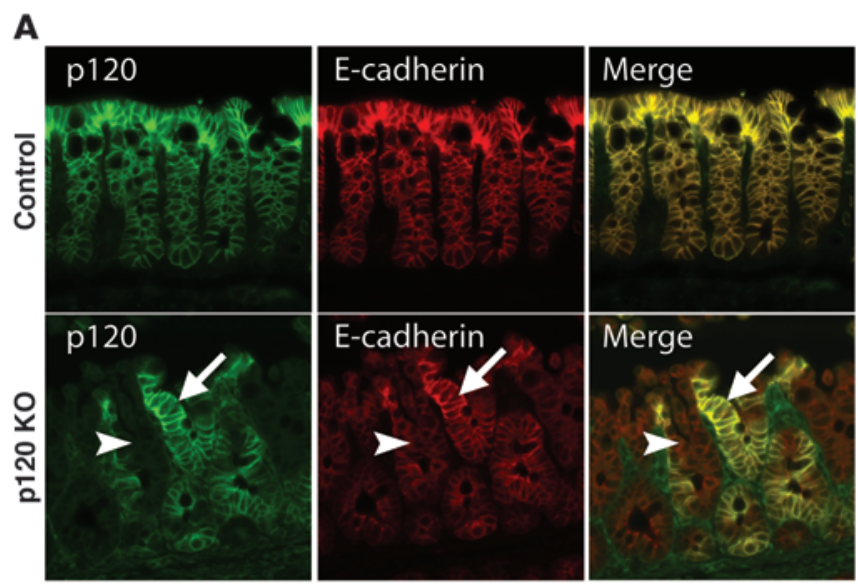

B
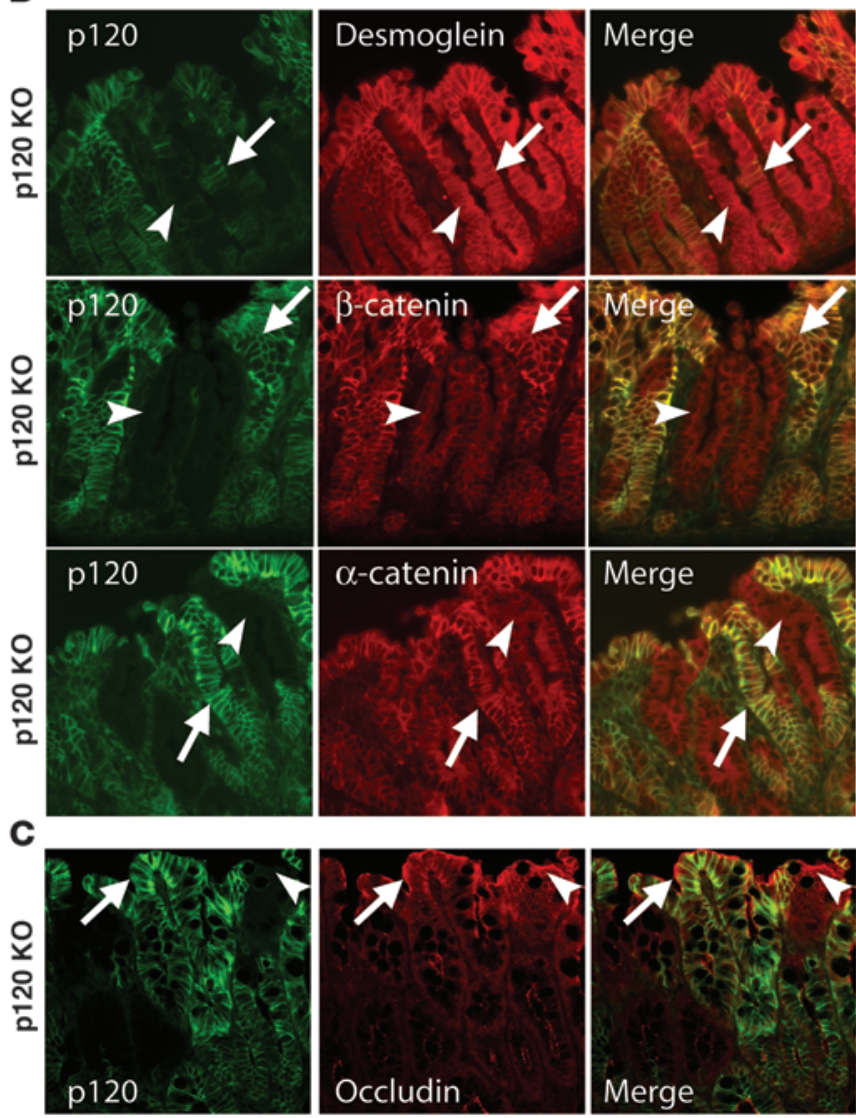

Figure 4

p120 ablation selectively downregulates the E-cadherin complex. Sections of 16-day-old colon were costained by immunofluorescence with antibodies to (A) p120 and E-cadherin, (B) p120 and desmoglein (top), $\beta$-catenin (middle), and $\alpha$-catenin (bottom), or (C) p120 and occludin. Mosaic presence and absence of p120 in the p120 KO panels allows direct contrast of effects in the same section. Arrows indicate p120 WT, and arrowheads indicate p120 KO. Note that desmoglein and occludin are not affected by p120 loss. Original magnification, $\times 20$.

bers in control and p120 KO colons were not significantly different $(P=0.14)$ (Figure 7A, right panels). Neutrophil infiltration is characteristic of ulcerative colitis and is generally associated with early acute inflammation (37).

COX-2 expression is increased in most active human IBD samples (40) and in mouse models of IBD (41). Therefore, we immunostained p120 control and KO tissues with antibodies to COX-2. Surprisingly, COX-2 staining in the epithelium was not noticeably altered and generally absent. Instead, we observed a striking and selective accumulation of a different COX-2-positive cell type that accumulated selectively in p120 KO areas of the colon (Figure 7B). Coimmunofluorescence staining for COX-2 and various other immune cell markers revealed that the vast majority of these COX-2-expressing cells were neutrophils (Figure 7C). Although neutrophil recruitment in this case may be due to exposure of the lamina propria to luminal antigens, we previously observed a similar phenomenon after p120 $\mathrm{KO}$ in the embryonic salivary gland, despite the sterile environment of the womb (A.B. Reynolds, unpublished observations). Thus, selective neutrophil recruitment to these areas could potentially reflect other cell autonomous consequences of p120 ablation.

Barrier defects versus inflammation. To clarify the role of the epithelial barrier, as apposed to other events linked to p120 ablation (e.g., neutrophil behavior), we generated p120 knockdown (KD)
HCA7 cells and evaluated the in vitro effect of p120 KD on several relevant phenotypes (Figure 8). WT (pLentiLox-GFP [pLL-GFP]) and p120 KD (pLentiLox-hp120i-GFP [pLL-hp120i-GFP]) HCA7 cells were plated on neutrophil permeable $(3-\mu \mathrm{m}$ pore size) filters, as described by Edens et al. (42). Neutrophils were then added to the basal side with chemoattractant (fMLP) on the apical side. The effects of $\mathrm{p} 120$ presence or absence on transepithelial electrical resistance (TER), neutrophil transmigration, and neutrophil attachment to the HCA7 monolayers were measured (Figure 8).

First, we assessed the effects of p120 ablation on neutrophil transmigration and TER of the epithelial monolayer. The TER of WT monolayers decreased dramatically upon addition of neutrophils (Figure 8A), as described previously (42). Interestingly, although respective WT and KD HCA7 monolayers were morphologically similar by light microscopy, TER was severely reduced by $120 \mathrm{KD}$, irrespective of neutrophil presence (Figure 8A). Neutrophil transmigration, on the other hand, was largely unaffected by p120 KD (Figure 8C).

The effect of p120 KD on TER was not unique to HCA7 cells, as similar effects were observed in p120 KD CaCo2 and HCT8 cells (data not shown). In general, TER in these p120 KD cell lines ranged from as low as 5\% (as compared with WT counterparts) (e.g., Figure 8A), to a high of $30 \%$, with most falling somewhere 
A
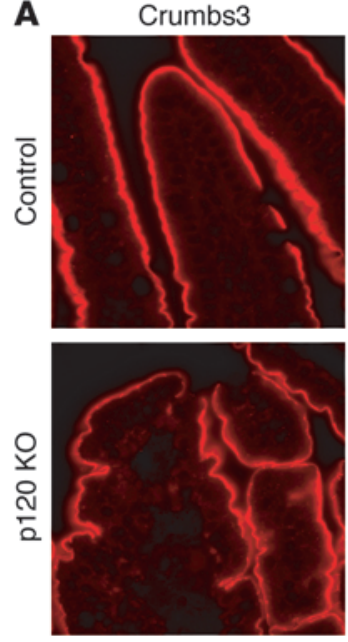

B Control

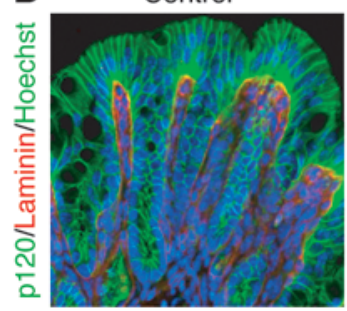

Figure 5

Apical, basolateral, and basement membrane markers are not directly altered by p120 ablation. Sections of WT or p120 KO intestine (A) or colon (B) were double stained by immunofluorescence with antibodies to apical (Crumbs3) and basolateral ( $\mathrm{Na} / \mathrm{K}$ ATPase) markers or with antibodies to the basement membrane marker (laminin) and the AJ marker (p120), respectively. Despite significant barrier defects and over $50 \%$ downregulation of $\mathrm{E}$-cadherin, $\mathrm{Na} / \mathrm{K}$ ATPase localization and polarity itself is not directly compromised. Note that patches of 120 loss similar to those in $\mathbf{B}$ are also present in the p120 KO samples in A. Original magnification, $\times 63(\mathbf{A}) ; \times 20(\mathbf{B})$.

in between. Although tight junctions are known to be the main effectors of barrier function, Figure 8B shows that ZO-1 staining was largely unaffected by p120 KD (compare control and KD panels). This observation is consistent with other evidence from Figures 3 and 4. Collectively, these data indicate that the tight junctions are largely intact, despite the consistently low levels of TER observed in P120 KD monolayers.

Next, we addressed the role of HCA7 p120 KD in modulating neutrophil attachment to polarized HCA7 cell monolayers (Figure 8D). Interestingly, p $120 \mathrm{KD}$ increased neutrophil attachment by 30 fold (Figure 8F), suggesting that p120 deficiency by itself induces changes that can be detected by neutrophils. We then examined the possibility that increased binding was caused by a soluble factor selectively produced by p120 KD cells. Conditioned media from WT and p120 KD cells was applied to the apical compartment of the WT cell monolayer and the experiments were repeated. However, neutrophil binding was unaffected by this parameter, suggesting that attachment is independent of secreted factors.

Notably, the neutrophils that accumulated in vivo in areas of p120 ablation were unusual in that they expressed very high levels of COX-2 (Figure 7, B and C), an enzyme closely associated with $\operatorname{IBD}(40,43)$ and colon cancer $(44)$. Therefore, we examined whether COX-2 levels were influenced by $\mathrm{p} 120 \mathrm{KD}$ in vitro. Neutrophils were introduced on the basal side of WT or p120-deficient
HCA7 cell monolayers and incubated for 2 or 18 hours (Figure 9A). At 2 hours, the number of neutrophils bound to $120 \mathrm{KD}$ cells was the same as at 18 hours, but little if any COX-2 expression was observed in either control (pLL-GFP) (data not shown) or p120 KD cells (pLL-hp120i-GFP) (Figure 9A). In contrast, at 18 hours, the vast majority of neutrophils bound to p120 KD cells were strongly COX-2 positive (Figure 9A). At this time, approximately $38 \%$ of neutrophils associated with WT cell monolayers expressed low levels of COX-2. Therefore, we separately quantified both high and low COX-2-expressing neutrophils to highlight the fact that strong COX-2 expression was almost exclusively confined to the population associated with p120 KD cells (Figure 9A, compare top and middle panels; quantified in Figure 9B, COX-2 high). To determine whether NF- $\kappa \mathrm{B}$ activation might be involved, we repeated the experiment and stained for NF- $\mathrm{KB}$. The result, however, was negative (data not shown), suggesting that COX-2 is upregulated by an alternative pathway in these cells. To control for this result, the same cells were stimulated with TNF- $\alpha$, which lead to obvious translocation of NF- $\mathrm{KB}$ to the nucleus (data not shown). Thus, neutrophil attachment and COX-2 upregulation is selectively increased by p120 KD in HCA7 cells.

\section{Discussion}

We show here that p120 function in the intestine is essential for epithelial homeostasis and indeed survival. In the absence of p120, the condition of the intestinal mucosa deteriorated rapidly over the first weeks of life and virtually all animals died by 3 weeks. The primary cause of death was massive intestinal bleeding, resulting from excessive cell sloughing and related defects in epithelial integrity throughout the small intestine. Although the early death of the animals precludes long-term studies in this model, it clearly illustrates the importance of p120 in the GI tract.

The severity of the phenotype is exacerbated by a significant inflammatory response, reminiscent of the major intestinal bowel diseases in humans. Previously, Jeffrey Gordon and colleagues showed that transgenic expression of DN-cadherin in the small intestine induced a profound Crohn-like IBD phenotype (45). Interestingly, of the approximately 17 described mouse models of IBD (46), the DN-cadherin mouse was the first of only a handful to date that clearly links the etiology to a physical defect in barrier function. Crohn-like characteristics in the DN-cadherin mouse included cell adhesion defects, neutrophil infiltration, and compensatory proliferation, all of which were central consequences of p120 ablation in our model. Although our phenotype was more severe, DN-cadherin expression in the Gordon experiments was also lethal initially and had to be scaled back by limiting the DN-cadherin ES cell contribution to the chimeric embryos used to generate these mice. Moreover, the expression of DN-cadherin was confined to the small intestine, whereas p120 ablation in our experiments was targeted to the entire intestinal tract and involved at least $50 \%$ of enterocytes. Given these differences, the phenotypes are in fact remarkably similar.

Although the Gordon experiments did not directly address the underlying mechanism of DN-cadherin action, they did docu- 
A
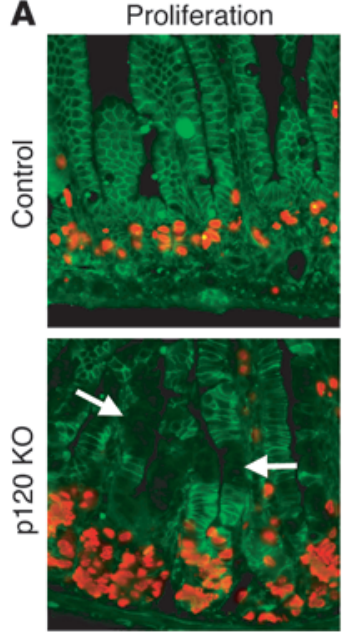

Migration
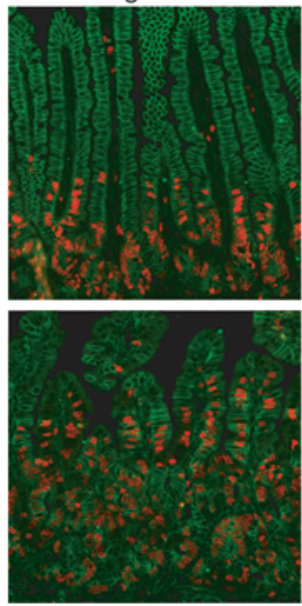

B
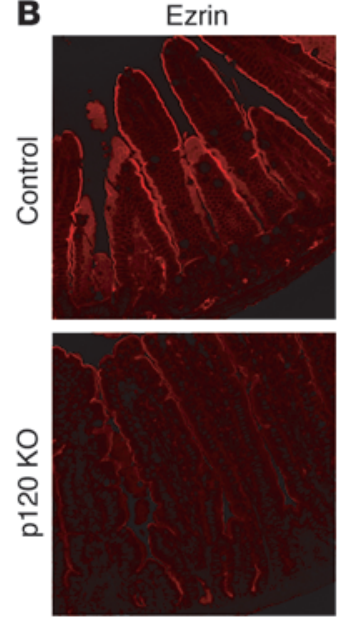

pERM
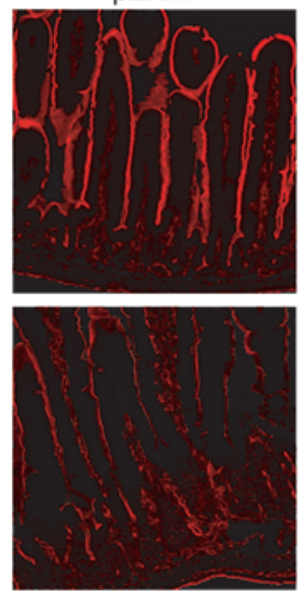

C
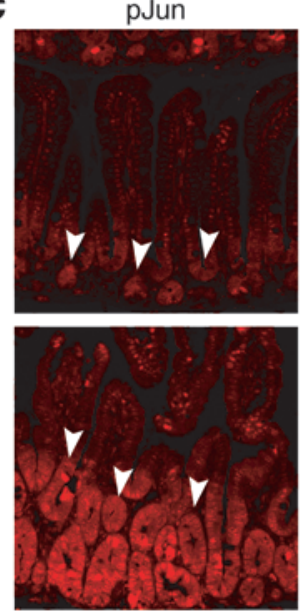

Figure 6

Inflammation-associated field effects in p120 KO tissue. (A) p120 KO and control 16-day-old mice were injected with BrdU, either 2 hours (proliferation) or 30 hours (migration) prior to sacrifice. Sections of the small intestine were costained with antibodies to BrdU (orange) and pp120 (green). (B) Sections of small intestine from WT and p120 KO mice were stained with antibodies to Ezrin or pERM. (C) Sections of small intestine from WT and p120 KO mice were stained with an antibody to phospho-Jun (pJun). Unlike changes in E-cadherin abundance, most other alterations were not restricted to mosaic patches of p120 ablation (e.g., arrows in A), and thus were not cell autonomous. Instead, most phenotypes were global (e.g., arrowheads in C), reflecting the type of widespread field effect frequently associated with inflammation. Original magnification, $\times 20$.

ment significant downregulation of endogenous E-cadherin (24, 47). DN-cadherin expression has, in fact, been used extensively to disrupt cell-cell adhesion in cultured cells and in vivo. The effect is characterized by rapid internalization and degradation of endogenous cadherins $(11,48-50)$, an effect also caused by removal of p120 $(10,11)$. Indeed, our work $(10,51)$ and evidence from the Kowalczyk laboratory (11) strongly suggest that DN-cadherin physically sequesters $\mathrm{p} 120$, thereby blocking its ability to bind and stabilize resident WT cadherins. Together, these observations strongly support the concept that DN-cadherins work primarily, if not entirely, via sequestration of $\mathrm{p} 120$. A potentially important difference between $\mathrm{p} 120 \mathrm{KD}$ and $\mathrm{DN}$-cadherin expression is that DN-cadherin can, in theory, bind up all p120 family members (e.g., $\delta$-catenin, p0071, ARVCF), whereas p120 ablation removes only p120. Indeed, the Gordon data show near complete loss of E-cadherin, whereas E-cadherin levels are reduced by approximately $50 \%$ in our model. With respect to p120 ablation in vivo, several lines of evidence suggest that redundant affects of family members account for the partial cadherin retention (52). While we cannot formally rule out other contributing factors, the defects in barrier function in both the DN-cadherin and in our p120 KO model likely reflect the critical role of p120 at adherens junctions. Thus, we believe that the consequences of removing p120 and overexpression of DN-cadherin are, in fact, mechanistically and functionally the same.

Although p120 has not been directly linked to IBD, several lines of evidence make p120 an interesting candidate with respect to etiology. First, the level of p120 in epithelial cells is probably the single most important factor in determining overall levels of classical and/or type II cadherins $(10,11,51)$. In many cultured epithelial cell lines, p120 KD almost completely eliminates cell-cell adhesion $(10,11)$, and here, we show in vivo and in vitro that p120 loss also manifests as a significant barrier defect. Second, recent evidence suggests that p120 is an important cell autonomous suppressor of inflammation (34). p120 KO in the epidermis has no discern- ible effect on cell-cell adhesion or barrier function, but nonetheless results in cell autonomous activation of NF- $\mathrm{KB}$ and a striking inflammatory response (34). Our data does not rule out such an effect in the intestines. Indeed, inflammation associated with the barrier defect may mask observation of cell autonomous effects, such as those observed in skin. In any event, it is interesting that the 2 most prominent phenotypes emanating from various $\mathrm{p} 120$ $\mathrm{KO}$ mice are also the main factors associated with the etiology of IBD and potentially relevant to the cycle of inflammation and barrier dysfunction that characterize the disease.

Interestingly, it has been reported that p120 is locally downregulated in $100 \%$ of active ulcerative colitis cases and $75 \%$ of active Crohn disease cases (53), the former being strongly associated with predisposition to colon cancer (i.e., colitis-associated cancer) (41). In addition, Kucharzik et al. have shown in IBD patients that E-cadherin and $\beta$-catenin are locally reduced in neutrophil-containing crypt abscesses but not in uninvolved areas (i.e., neutrophil negative), a result consistent with transient downregulation of p120 (54). Finally, neutrophil attachment to the p120-deficient HCA7 monolayer was elevated 30 fold and correlated with striking upregulation of COX-2, an event likely to increase $\mathrm{PGE}_{2}$ levels and exacerbate local inflammation.

Another critical consequence of p120-deficiency in HCA7 monolayers was the considerably reduced ability to establish TER. Despite the physical presence of tight junctions, their role in maintaining barrier function is probably indirectly compromised by major defects in the adherens junctions. One possibility is that tight junctions depend in part on architectural support provided by strong basolateral cell-cell adhesion. In any event, these data strongly suggest that the epithelial barrier in the p120-null intestine is inherently leaky from birth and most likely the initial defect in the progression to an inflammatory phenotype.

Of note, we observed a significant influx of neutrophils into p120-null areas of the colon, and there was a clear tendency for 
A Neutrophils
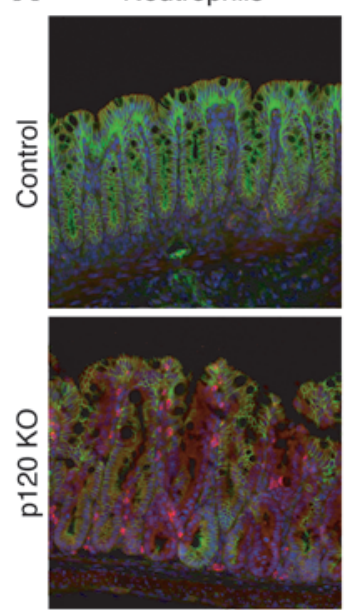

$\mathbf{B}$

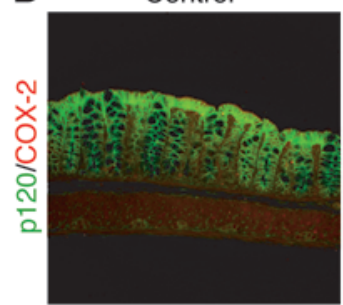

C

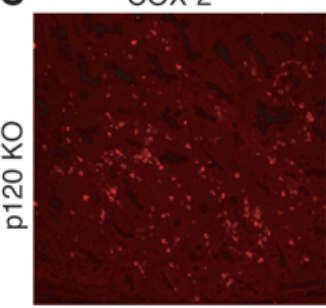

Macrophages
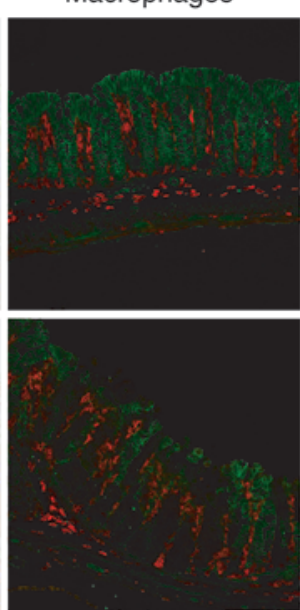
p120 KO

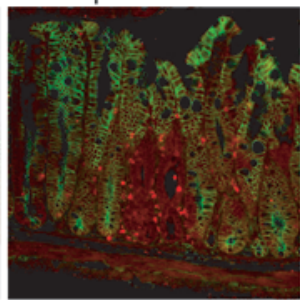

Neutrophils

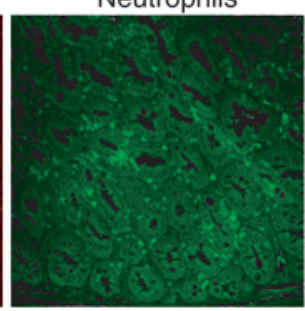

$\mathrm{CD}^{+} \mathrm{T}$ cells
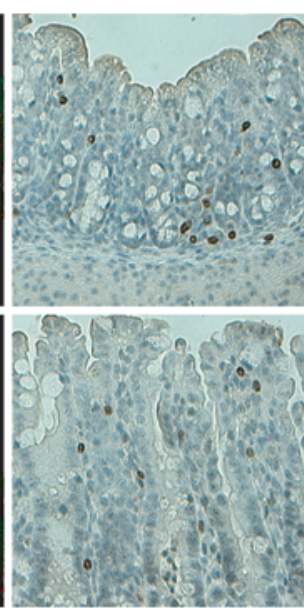

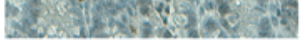

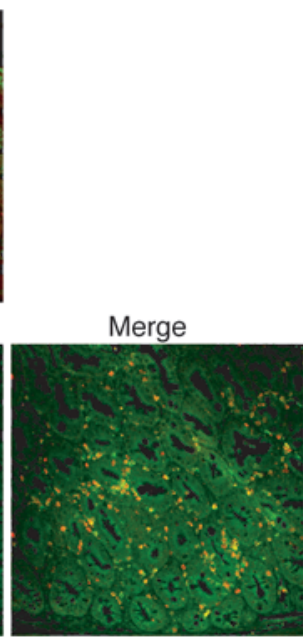

Figure 7

Selective association of COX-2-positive neutrophils with areas of $\mathrm{p} 120$ negativity. Sections of control and $\mathrm{p} 120 \mathrm{KO}$ colon from 16-day-old mice were costained with antibodies to (A) p120 and neutrophils (left), p120 and macrophages (middle), and p120 and $\mathrm{CD}^{+} \mathrm{T}$ cells (right), or (B) p120 and COX-2, or (C) COX-2 and neutrophils. Original magnification, $\times 20$.

them to concentrate in patches of p120 negativity (see Figure 7A). Surprisingly, neutrophil infiltrates were not observed in the small intestine, despite significant epithelial erosion and clear evidence of inflammation. Thus, p120 negativity is unlikely, by itself, to explain the selective accumulation of neutrophils in the p120 KO colon. In the DN-cadherin model, neutrophil infiltration of the small intestine was a prominent feature of the Crohn-like phenotype, but neutrophils were not evident at 6 weeks, despite several early indicators of disease (24). Therefore, adhesive defects in the DN-cadherin model clearly preceded neutrophil infiltration, which was not obvious until approximately 3 months. In contrast, all of our animals were dead by day 23 .

Another potentially relevant variable is the bacterial colonization of the intestine shortly after birth. For example, in human infants, the initially sterile intestine is fully colonized between 1 and 2 weeks of age, and this coincides temporally with the onset of necrotizing enterocolitis, an acute inflammatory disease that is

often lethal $(55,56)$. Notably, similar colonization rates have been reported in mice (57), and the disease progression in our mice takes place over a similar time frame. Indeed, although p120 ablation in our mice occurs well before birth, differences in villus morphology were not pronounced until approximately day 10 . Thus, it is likely that the rapid decline at this time point is linked to acceleration of the inflammatory response, due in part to the exponential accumulation of gut flora over this period.

Interpretation of cell-autonomous and -nonautonomous events were complicated by the ubiquitous presence of an inflammatory field affect. Whereas E-cadherin (and catenins) downregulation was clearly restricted to $\mathrm{p} 120$-null cells (Figure 4), the staining of most other markers was independent of p120 expression and instead appeared to be driven by widespread inflammation. For example, we originally stained WT and p120 KO tissue for both Ezrin and activated Ezrin (pERM) to look for possible p120-related effects on Rho GTPase activity $(38,58)$. However, although overall staining of WT and p120 KO tissue differed markedly (see Figure 6B), colocalization studies revealed no correlation with the clear mosaic pattern of p120 expression (as seen in Figure 6A). Instead, the differences caused by $120 \mathrm{KO}$ were reflected evenly throughout the tissue, as is frequently observed in the inflamed colon. Staining for activated Jun (pJun), as an indicator of stress pathway activation $(39,59)$, revealed similar results as early as day 5 (data not shown), consistent with an early defect in barrier function. Thus, most of the differences between WT and $\mathrm{KO}$ tissue were reflective of the inflammatory field effect rather than direct cell-autonomous alterations. However, we cannot completely rule out the possibility that the field effect itself is initiated by a cell-autonomous event, such as direct upregulation of inflammatory cytokines, as apposed to an indirect consequence of the barrier defect.

The increased cell proliferation observed in both the small intestine (Figure 6A) and colon (data not shown) may also be linked to inflammation (e.g., cytokine stimulation), as has been reported by others $(60,61)$, or alternatively, to compensatory mechanisms that respond to excessive cell damage and exfoliation $(30,47)$. Increased cell migration may be inflammation induced as well (62). However, it is known that increased levels of E-cadherin expression dramatically slow upward migration along the villus (45). Thus, p120 ablation could have the opposite effect, consistent with a cell-autonomous mechanism. It is likely that all of these factors contribute to the change in migration.

Whether intestinal inflammation following p120 KO is influenced by NF- $\kappa \mathrm{B}$ activity, as reported previously in the epidermis $(34,63)$, is not yet clear. p120 KD in vitro in the colon carcinoma cell line HCA7 did not increase NF-кB activity (data not shown). However, COX-2 expression, which is sometimes activated by NF- $\kappa \mathrm{B}$, was markedly elevated in neutrophils exposed to p120 $\mathrm{KD} \mathrm{HCA} 7$ cells. Of note, most neutrophils in the p120 KO colon were also COX-2 positive. Elevated COX-2 activity is thought to play an important role in both IBD and colon cancer $(41,43,44)$, but the source of the activity is controversial. Elevated COX-2 has been reported variously in epithelial cells, as well as almost every stromal cell type $(44,64)$ - macrophages in particular (65). Intestinal COX-2 upregulation has been described in neutrophils as well 
A Transepithelial electrical resistance

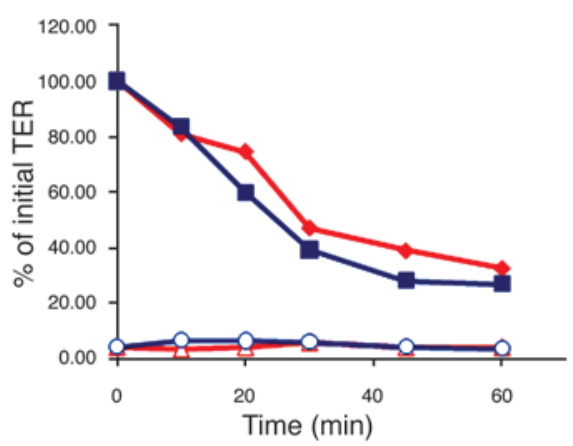

$\mathbf{C}$

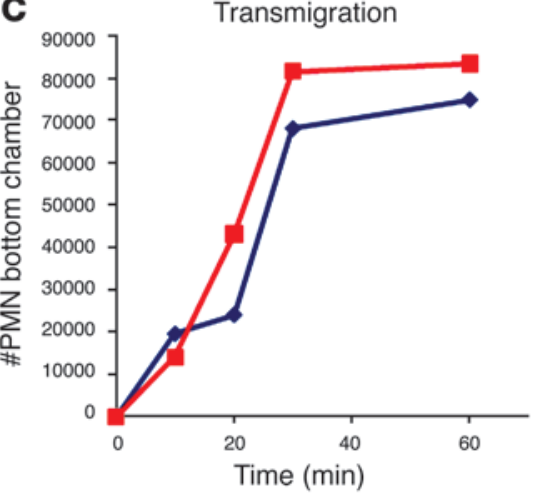

$\mathbf{F}$

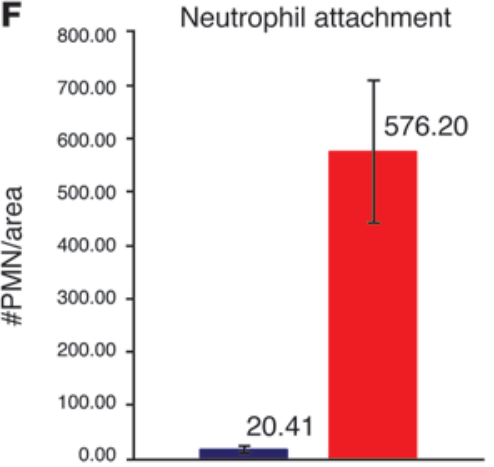

B

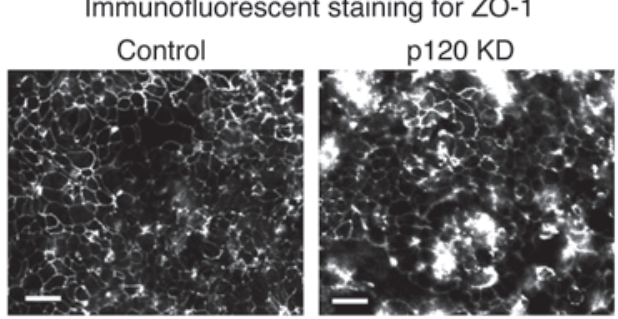

D

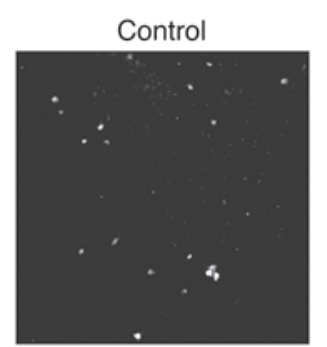

E

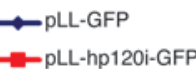

- \#PMN/attached to PLL-GFP monolayer

- \#PMN/attached to PLL-hp120i-GFP monolayer

Figure 8

p120 KD in vitro in HCA7 cells abolishes barrier function and increases neutrophil attachment. (A) TER was measured over time during neutrophil migration (with and without fMLP) across control (pLL-GFP) and p120-deficient (pLL-hp120i-GFP) HCA7 monolayers. TER was measured in Ohms and normalized to reflect the fraction of total TER generated by control HCA7 monolayers at time 0 (before addition of neutrophils). TER was very low or absent in monolayers generated with p120-deficient HCA7 cells. med, media. (B) To visualize tight junctions, the control and p120-deficient HCA7 monolayers used to generate the TER data in A were immunostained with an antibody directed against ZO-1. Scale bar: $20 \mu \mathrm{m}$. (C) Neutrophil transmigration (with $\mathrm{FMLP}$ ) was measured on WT and p120-deficient HCA7 monolayers. Neutrophil transmigration was unaffected by $\mathrm{p} 120 \mathrm{KD}$. (D-F) The experiment in $\mathbf{C}$ was repeated with an incubation time of 2 hours. Filters were washed vigorously and neutrophil attachment to WT HCA7 cells (D) or p120-deficient HCA7 cells (E) was assayed by direct immunofluorescent staining. The results are quantified in F. Neutrophil attachment to the p120-deficient HCA7 monolayer was increased 30 fold. Data represent mean \pm SD. Original magnification, $\times 20(B, D$, and E).

$(43,66)$ but is less common. Thus, p120 ablation in enterocytes could potentially promote inflammation, in part, by inducing neutrophil attachment and subsequent upregulation of COX-2.

Irrespective of NF- $\kappa \mathrm{B}$, it is clear that $\mathrm{p} 120$ ablation in intestinal cell lines has marked effects on neutrophil behavior. Attachment of neutrophils to cultured HCA7 cells was dramatically elevated when p120 levels were reduced. This effect does not appear to be caused by soluble factors, because conditioned media from p120-deficient cells did not influence neutrophil binding to their p120-positive counterparts.
Thus, one explanation is that p120 ablation itself directly induces the upregulation or exposure of one or more neutrophil binding factors. Alternatively, increased neutrophil attachment could be an indirect consequence of p120 downregulation, if the mechanism is driven primarily by loss of barrier function and/or abnormal exposure of basolateral ligands. Either way, p120 loss causes increased physical, and probably functional, epithelial interactions with neutrophils.

In summary, we show that p120 is essential for epithelial homeostasis in the intestine. The effects of p120 ablation are, in fact, 
A
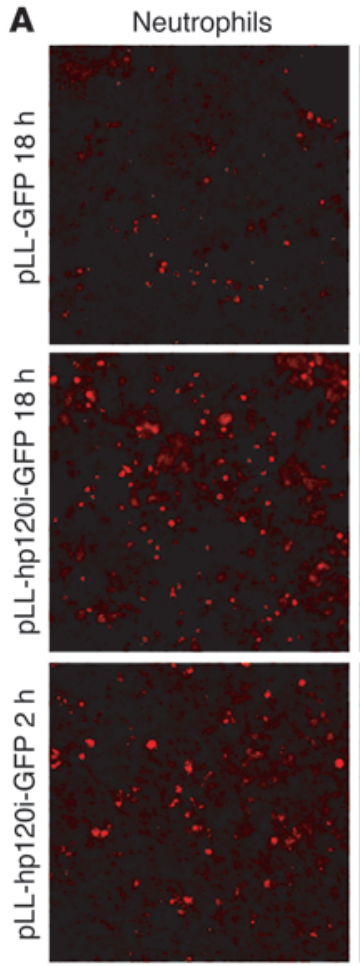

B

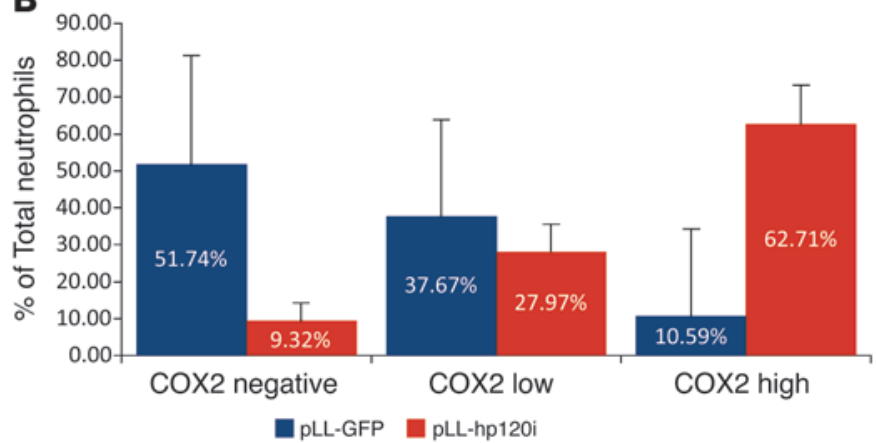

\section{Figure 9}

COX-2 is upregulated in neutrophils after exposure to p120-deficient HCA7 monolayers. (A) Neutrophil transmigration experiments were conducted as described in Figure 5, except that the incubation time was either 2 hours or 18 hours. After vigorous washing, filters were costained for myeloperoxidase (neutrophils) and COX-2. As also observed at 2 hours (Figure 5), neutrophil attachment to p120-deficient monolayers (pLL-hp120i-GFP) was substantially increased relative to that of control (pLL-GFP). However, there is no upregulation of COX-2 at 2 hours (bottom middle panel). Original magnification, $\times 20$. (B) Quantification of neutrophil attachment and COX-2 upregulation at 18 hours Neutrophils were divided into 3 groups based on COX-2 staining intensity. Neutrophils with COX-2 staining lower than that of background levels $(18 \%$ of maximum intensity) were considered COX-2 negative, neutrophils with COX-2 staining between $18 \%$ and $35 \%$ of maximum intensity were considered COX-2 low, and neutrophils with higher than $35 \%$ of maximum COX-2 intensity were considered COX-2 high. We found that $62.71 \%$ of the neutrophils attached to p120-deficient monolayers have high COX-2 staining. In contrast, only $10.59 \%$ of the neutrophils attached to WT monolayers have high COX-2 staining. Data represent mean \pm SD. similar to the IBD phenotype described previously in mice overexpressing DN-cadherin (24). Importantly, it is increasingly clear that DN-cadherins sequester p120, thereby inducing internalization and destruction of endogenous cadherins (52). Thus, we suggest that $\mathrm{p} 120$ downregulation and DN-cadherin expression are mechanistically and functionally equivalent. Our in vitro data illustrate a critical role for p120 in maintaining barrier function. However, the data also reveal a striking role for p120 in modulating neutrophil attachment and probably activation. Whether these roles contribute directly to the etiology of human IBD or adenoma formation in mice, as implied previously by the DN-cadherin data, is not yet known. Importantly, identification of p120 as a central effector of the DN-cadherin phenotype provides not only mechanism but also an actionable target for potential therapeutic intervention. An important next step is to generate inducible p120 KO models to control the level of disease and permit long-term studies. Indeed, in addition to inflammatory disease, the DN-cadherin mice develop adenomas at approximately 6 months. Together, these observations suggest that long-term p120 loss or inactivation will be tumorigenic.

\section{Methods}

Mice. All experiments involving animals were approved by the Vanderbilt University Institutional Animal Care and Use Committee. Mice were maintained under a strict 12-hour-light/dark cycle, with free access to chow and water. Mice containing the floxed p120 allele were generated, as described previously, by our laboratory (32). $\mathrm{p} 120^{\mathrm{F} / \mathrm{F}}$ mice were crossed with villin-Cre mice (35) to target p120 ablation to the small intestine and colon. Littermate controls were used for all experiments to control for the mixed background of these mice.

To obtain blood samples for hematocrit analyses, terminal heart punctures were performed on 16-day-old mice. Blood was collected using heparin-coated capillary tubes. Serum was separated by centrifugation, and packed red blood cell volume was measured. To determine proliferation and migration rates, mice were injected intraperitoneally with BrdU, either 2 hours or 30 hours before sacrificing, respectively.

Immunobistochemistry. Swiss-rolled intestinal tissue was fixed in formalin overnight at $4{ }^{\circ} \mathrm{C}$ and then embedded in paraffin. Paraffin-embedded sections $(5 \mu \mathrm{m})$ were prepared for $\mathrm{H} \& \mathrm{E}$ staining and immunohistochemistry (IHC). Sodium citrate antigen retrieval was used for all antibodies, except for anti-F4/80 and anti-BrdU. Antigen retrieval for anti-F4/80 was achieved by incubating sections in trypsin at $37^{\circ} \mathrm{C}$. For fluorescence IHC, samples 
were incubated with primary and secondary antibodies overnight at $4{ }^{\circ} \mathrm{C}$ and for 2 hours at room temperature, respectively.

The following primary antibodies were used: $\mathrm{F} 1 \alpha \mathrm{SH}$, an affinity-purified rabbit anti-p120 polyclonal antibody, was generated by the Reynolds laboratory; pp120 (catalog 610133) and anti-E-cadherin (catalog 610181) were from BD Biosciences - Transduction Laboratories; anti- $\beta$-catenin (catalog C2206) and anti- $\alpha$-catenin (catalog C2066) were from Sigma-Aldrich; $\mathrm{Na}^{+}$, $\mathrm{K}^{+}$ATPase (catalog 05-369) was from Upstate Biotechnology; Desmoglein 1 (catalog sc-20114) was from Santa Cruz Biotechnology Inc.; BrdU (cata$\log$ OBT0030) was from Accurate Chemical \& Scientific Corp.; rat antimouse neutrophils (catalog MCA771GA) and rat anti-mouse F4/80 (catalog MCA497R) were from Serotec; anti-laminin (catalog L9393) was from Sigma-Aldrich; anti-phospho-ERM (catalog 31415) was from Cell Signaling Technology; anti-phospho-c-Jun (catalog 9164) was from New England Biolabs; anti-Ezrin (catalog 3C12) was from NeoMarkers; COX-2 polyclonal antibody was from Caymen Chemical; anti-ZO-1 (catalog 40-2200) was from Invitrogen; and Crumbs 3 was a gift from Ben Margolis (University of Michigan Medical School). Secondary antibodies, conjugated to either the 488 Alexa Fluor or the 594 Alexa Fluor, were obtained from Molecular Probes.

$E M$. Small and large intestine samples were fixed in $2.5 \%$ glutaraldehyde/ $0.1 \mathrm{~mol} / 1$ sodium cacodylate (glutaraldehyde was from Electron Microscopy Sciences). After desired regions were located on a semithin section, 70-nm sections were placed on slot grids, stained with uranyl acetate and lead citrate, and analyzed by transmission EM (Philips CM-12 electron microscope).

Cell lines, reagents, and constructs. HCA7 cells were grown in DME supplemented with $10 \% \mathrm{FBS}, 100 \mathrm{U} / \mathrm{ml}$ penicillin, and $100 \mathrm{mg} / \mathrm{ml}$ streptomycin. pLL lentiviral vectors expressing shRNA directed against human p120 were generated by cloning annealed sense (tgccagaggtggttcggatattcaagagatatccgaaccacctctggcttttttc) and anti-sense (tcgagaaaaaagccagaggtggttcggatatctcttgaatatccgaaccacctctggca) oligos into the pLL 5.0 vector with $\mathrm{HpaI} / \mathrm{XhoI}$ restriction sites. Production of pLL virus for shRNA expression was conducted in HEK293T cells, as described previously (67).

TER. TER was measured in ohms as described previously (66). In WT cells, all the resistance measurements were normalized to a percentage of the TER at time point 0 , which was considered to be $100 \%$. The TER for p120 KD cells was normalized in a similar manner but was also multiplied by a coefficient equal to the ratio between WT and P120 KD TER at time point 0 .

Polymorphonuclear leukocytes transepithelial migration. Normal human polymorphonuclear leukocytes (PMNs) were isolated, as described previously by Edens et al. (42). PMN transmigration experiments were performed as previously described in detail (68). Briefly, inserts were washed 2 times with PBS, placed into new wells containing HBSS (Sigma-Aldrich), and allowed to equilibrate for 15 minutes. Purified PMNs were added to the upper reservoir to a final density of $3.5 \times 10^{6} \mathrm{cell} / \mathrm{cm}^{2}$. PMN migration was stimulated with a $1 \mathrm{mM}$ gradient of $\mathrm{mLLP}$.

After a defined period of PMN transepithelial migration, HCA7 monolayers were washed several times with PBS to remove nonadherent or semiadherent PMNs. For immunofluorescence analysis, monolayers were fixed with $3 \%$ PFA for 30 minutes. Monolayers were then permeabilized with $0.2 \%$ Triton X-100 for 15 minutes. Cell were blocked in 5\% milk to reduce nonspecific staining for 1 hour. Samples were incubated with primary antibodies for 1.5 hours at room temperature and then probed with secondary antibodies coupled with Alexa Fluor 594 or Alexa Fluor 647 (Molecular Probes) for 1 hour at room temperature. Cells were stained with Hoechst dye to visualize the nuclei. Stained monolayers were mounted in Prolong Gold Mounting Media (Invitrogen).

The PMN content of the lower reservoir was quantified by assaying for myeloperoxidase. Myeloperoxidase from the lower reservoir was released by addition of Triton X-100 to a final concentration of $0.5 \%$. For each sample, color development was assayed at $405 \mathrm{~nm}$ on a plate reader, after mixing equal volumes of the sample and a 2,2'-azino-bis(3-ethylbenzthiazoline6-sulfonic acid) liquid substrate system for ELISA (catalog A3219; SigmaAldrich). After appropriate color development, the reaction was stopped by adding SDS to a final concentration of $0.5 \%$. The assay was standardized with the known concentrations of the same PMNs used in each experiment.

PMNCOX-2 quantification. PMN transepithelial migration experiments were performed as described above, except that neutrophils were incubated with HCA7 monolayers for either 2 or 18 hours. Neutrophils were divided into 3 groups based on COX-2 staining intensity. Neutrophils with COX-2 staining lower than that of background levels (18\% of maximum intensity) were considered COX-2 negative, neutrophils with COX-2 staining between $18 \%$ and $35 \%$ of maximum intensity were considered COX- 2 low, and neutrophils with higher than $35 \%$ of maximum COX-2 intensity were considered COX-2 high.

Microscopy. Fluorescence images were taken on an Axioplan 2 microscope (Carl Zeiss Inc.) with a Hamamatsu ORCA-ER digital camera. Images were analyzed and processed with OpenLab (PerkinElmer) and MetaMorph (MDS Analytical Technologies) software. Adobe Photoshop and Illustrator were used to generate figures.

Statistics. For calculations to determine statistical significance, the Student's $t$ test and ANOVA were used. $P$ values of less than 0.05 were considered significant. All calculations were performed using Microsoft Excel 2004 for Mac.

\section{Acknowledgments}

We wish to acknowledge the outstanding assistance of several Vanderbilt Core facilities, including the Human Tissue Acquisition Resource, the Cell Imaging Shared Resource, and the Antibody Shared Resource, and the expert technical assistance of Nichole Lobdell. This work was supported by the Vanderbilt Cancer Center Support grant (P30-CA068485), the National Cancer Institute (NCI) grant RO1 CA111947, and the Vanderbilt GI SPORE (50 CA95103). Andrey Efimov and Michael Davis were supported in part by NCI grant RO1 CA055724.

Received for publication October 13, 2009, and accepted in revised form March 24, 2010.

Address correspondence to: Albert B. Reynolds, Vanderbilt University Medical Center, 771 PRB, 2220 Pierce Ave., Nashville, Tennessee 37232, USA. Phone: 615.343.9532; Fax: 615.936.6399; E-mail: al.reynolds@vanderbilt.edu.
1. Yap AS. The morphogenetic role of cadherin cell adhesion molecules in human cancer: a thematic review. Cancer Invest. 1998;16(4):252-261.

2. Takeichi M. Morphogenetic roles of classic cadherins. Curr Opin Cell Biol. 1995;7(5):619-627.

3. Oka $\mathrm{H}$, et al. Immunohistochemical evaluation of E-cadherin adhesion molecule expression in human gastric cancer. Virchows Arch A Pathol Anat Histopathol. 1992;421(2):149-156.

4. Berx G, etal.E-cadherin is a tumour/invasion suppressor gene mutated in human lobular breast cancers. EMBO J. 1995;14(24):6107-6115.
5. Berx G, et al. E-cadherin is inactivated in a majority of invasive human lobular breast cancers by truncation mutations throughout its extracellular domain. Oncogene. 1996;13(9):1919-1925.

6. Beavon IR. The E-cadherin-catenin complex in tumour metastasis: structure, function and regulation. Eur J Cancer. 2000;36(13 Spec No):1607-1620.

7. Herrenknecht K, Ozawa M, Eckerskorn C, Lottspeich F, Lenter M, Kemler R. The uvomorulin-anchorage protein alpha catenin is a vinculin homologue. Proc Natl Acad Sci U S A. 1991;88(20):9156-9160.
8. Nagafuchi A, Takeichi M, Tsukita S. The $102 \mathrm{kd}$ cadherin-associated protein: similarity to vinculin and posttranscriptional regulation of expression. Cell. 1991;65(5):849-857.

9. Rimm DL, Koslov ER, Kebriaei P, Cianci CD, Morrow JS. Alpha 1(E)-catenin is an actin-binding and -bundling protein mediating the attachment of F-actin to the membrane adhesion complex. Proc Natl Acad Sci U S A. 1995;92(19):8813-8817.

10. Davis MA, Ireton RC, Reynolds AB. A core function for p120-catenin in cadherin turnover. J Cell Biol. 2003;163(3):525-534. 
11. Xiao K, et al. Cellular levels of p120 catenin function as a set point for cadherin expression levels in microvascular endothelial cells. J Cell Biol. 2003;163(3):535-545

12. Wildenberg GA, et al. p120-catenin and p190RhoGAP regulate cell-cell adhesion by coordinating antagonism between Rac and Rho. Cell. 2006;127(5):1027-1039.

13. Anastasiadis PZ, et al. Inhibition of RhoA by $\mathrm{p} 120$ catenin. Nat Cell Biol. 2000;2(9):637-644.

14. Noren NK, Liu BP, Burridge K, Kreft B. p120 catenin regulates the actin cytoskeleton via Rho family GTPases. J Cell Biol. 2000;150(3):567-580.

15. Grosheva I, Shtutman M, Elbaum M, Bershadsky AD. p120 catenin affects cell motility via modulation of activity of Rho - family GTPases: a link between cell-cell contact formation and regulation of cell locomotion. J Cell Sci. 2001;114(pt 4):695-707.

16. Daniel JM, Reynolds AB. The catenin p120(ctn) interacts with Kaiso, a novel BTB/POZ domain zinc finger transcription factor. Mol Cell Biol. 1999;19(5):3614-3623.

17. Prokhortchouk A, et al. The p120 catenin partner Kaiso is a DNA methylation-dependent transcriptional repressor. Genes Dev. 2001;15(13):1613-1618.

18. Daniel JM. Dancing in and out of the nucleus: p120(ctn) and the transcription factor Kaiso. Biochim Biophys Acta. 2007;1773(1):59-68.

19. Kim SW, et al. Non-canonical Wnt signals are modulated by the Kaiso transcriptional repressor and p120-catenin. Nat Cell Biol. 2004;6(12):1212-1220.

20. Park JI, et al. Kaiso/p120-catenin and TCF/betacatenin complexes coordinately regulate canonical Wht gene targets. Dev Cell. 2005;8(6):843-854.

21. Lopes EC, et al. Kaiso contributes to DNA methylation-dependent silencing of tumor suppressor genes in colon cancer cell lines. Cancer Res. 2008;68(18):7258-7263.

22. Spring CM, Kelly KF, O’Kelly I, Graham M, Crawford HC, Daniel JM. The catenin p120ctn inhibits Kaiso-mediated transcriptional repression of the beta-catenin/TCF target gene matrilysin. Exp Cell Res. 2005;305(2):253-265.

23. Ruzov A, et al. The interaction of xKaiso with xTcf3: a revised model for integration of epigenetic and Wnt signalling pathways. Development. 2009;136(5):723-727.

24. Hermiston ML, Gordon JI. Inflammatory bowel disease and adenomas in mice expressing a dominant negative $\mathrm{N}$-cadherin. Science. 1995;270(5239):1203-1207.

25. Loftus EV Jr. Clinical epidemiology of inflammatory bowel disease: Incidence, prevalence, and environmental influences. Gastroenterology. 2004;126(6):1504-1517.

26. Goyette P, Labbe C, Trinh TT, Xavier RJ, Rioux JD. Molecular pathogenesis of inflammatory bowel disease: genotypes, phenotypes and personalized medicine. Ann Med. 2007;39(3):177-199.

27. Fiocchi C. Inflammatory bowel disease: etiology and pathogenesis. Gastroenterology. 1998;115(1):182-205.

28. Olson TS, et al. The primary defect in experimental ileitis originates from a nonhematopoietic source. J Exp Med. 2006;203(3):541-552.

29. Laukoetter MG, et al. JAM-A regulates permeability and inflammation in the intestine in vivo. $J$ Exp Med. 2007;204(13):3067-3076.

30. Williams IR, Parkos CA. Colonic neutrophils in inflammatory bowel disease: double-edged swords of the innate immune system with protective and destructive capacity. Gastroenterology. 2007;133(6):2049-2052.

31. Schnoor M, Parkos CA. Disassembly of endothelial and epithelial junctions during leukocyte transmigration. Front Biosci. 2008;13:6638-6652.

32. Davis MA, Reynolds AB. Blocked acinar development, e-cadherin reduction, and intraepithelial neoplasia upon ablation of p120-catenin in the mouse salivary gland. Dev Cell. 2006;10(1):21-31.

33. Elia LP, Yamamoto M, Zang K, Reichardt LF. p120 catenin regulates dendritic spine and synapse development through Rho-family GTPases and cadherins. Neuron. 2006;51(1):43-56

34. Perez-Moreno M, Davis MA, Wong E, Pasolli HA, Reynolds AB, Fuchs E. p120-catenin mediates inflammatory responses in the skin. Cell. 2006;124(3):631-644.

35. Madison BB, Dunbar L, Qiao XT, Braunstein K, Braunstein E, Gumucio DL. Cis elements of the villin gene control expression in restricted domains of the vertical (crypt) and horizontal (duodenum, cecum) axes of the intestine. J Biol Chem. 2002;277(36):33275-33283.

36. Maunoury R, Robine S, Pringault E, Leonard N, Gaillard JA, Louvard D. Developmental regulation of villin gene expression in the epithelial cell lineages of mouse digestive and urogenital tracts. Development. 1992;115(3):717-728.

37. Xavier RJ, Podolsky DK. Unravelling the pathogenesis of inflammatory bowel disease. Nature. 2007;448(7152):427-434.

38. Saotome I, Curto M, McClatchey AI. Ezrin is essential for epithelial organization and villus morphogenesis in the developing intestine. Dev Cell. 2004;6(6):855-864

39. Johnson GL, Nakamura K. The c-jun kinase/ stress-activated pathway: regulation, function and role in human disease. Biochim Biophys Acta. 2007;1773(8):1341-1348.

40. Jupp J, et al. Colonic expression of leukotrienepathway enzymes in inflammatory bowel diseases. Inflamm Bowel Dis. 2007;13(5):537-546.

41. Hegazi RA, Saad RS, Mady H, Matarese LE, O’Keefe S, Kandil HM. Dietary fatty acids modulate chronic colitis, colitis-associated colon neoplasia and COX2 expression in IL-10 knockout mice. Nutrition. 2006;22(3):275-282.

42. Edens HA, et al. Neutrophil transepithelial migration: evidence for sequential, contact-dependent signaling events and enhanced paracellular permeability independent of transjunctional migration. JImmunol. 2002;169(1):476-486.

43. Popivanova BK, et al. Blocking TNF-alpha in mice reduces colorectal carcinogenesis associated with chronic colitis. J Clin Invest. 2008;118(2):560-570.

44. Wendum D, Masliah J, Trugnan G, Flejou JF. Cyclooxygenase- 2 and its role in colorectal cancer development. Virchows Arch. 2004;445(4):327-333.

45. Hermiston ML, Wong MH, Gordon JI. Forced expression of E-cadherin in the mouse intestinal epithelium slows cell migration and provides evidence for nonautonomous regulation of cell fate in a selfrenewing system. Genes Dev. 1996;10(8):985-996.

46. Wirtz S, Neurath MF. Mouse models of inflammatory bowel disease. Adv Drug Deliv Rev. 2007;59(11):1073-1083

47. Hermiston ML, Gordon JI. In vivo analysis of cadherin function in the mouse intestinal epithelium: essential roles in adhesion, maintenance of differentiation, and regulation of programmed cell death. J Cell Biol. 1995;129(2):489-506.

48. Troxell ML, Chen YT, Cobb N, Nelson WJ, Marrs JA. Cadherin function in junctional complex rearrangement and posttranslational control of cadherin expression. Am J Physiol. 1999; 276(2 pt 1):C404-418.

49. Nieman MT, Kim JB, Johnson KR, Wheelock
MJ. Mechanism of extracellular domain-deleted dominant negative cadherins. J Cell Sci. 1999; 112(pt 10):1621-1632.

50. Zhu AJ, Watt FM. Expression of a dominant negative cadherin mutant inhibits proliferation and stimulates terminal differentiation of human epidermal keratinocytes. J Cell Sci. 1996; 109(pt 13):3013-3023.

51. Ireton RC, et al. A novel role for p120 catenin in Ecadherin function. J Cell Biol. 2002;159(3):465-476.

52. Reynolds AB, Carnahan RH. Regulation of cadherin stability and turnover by $120 \mathrm{ctn}$ : implications in disease and cancer. Semin Cell Dev Biol. 2004;15(6):657-663.

53. Karayiannakis AJ, et al. Expression of catenins and Ecadherin during epithelial restitution in inflammatory bowel disease. J Pathol. 1998;185(4):413-418.

54. Kucharzik T, Walsh SV, Chen J, Parkos CA, Nusrat A. Neutrophil transmigration in inflammatory bowel disease is associated with differential expression of epithelial intercellular junction proteins. Am J Pathol. 2001;159(6):2001-2009.

55. Lin PW, Nasr TR, Stoll BJ. Necrotizing enterocolitis: recent scientific advances in pathophysiology and prevention. Semin Perinatol. 2008;32(2):70-82.

56. Sodhi C, Richardson W, Gribar S, Hackam DJ. The development of animal models for the study of necrotizing enterocolitis. Dis Model Mech. 2008;1(2-3):94-98.

57. Schaedler RW, Dubos R, Costello R. The development of the bacterial flora in the gastrointestinal tract of mice. J Exp Med. 1965;122:59-66.

58. Curto M, McClatchey AI. Ezrin...a metastatic detERMinant? Cancer Cell. 2004;5(2):113-114.

59. Leppa S, Bohmann D. Diverse functions of JNK signaling and c-Jun in stress response and apoptosis. Oncogene. 1999;18(45):6158-6162.

60. Mizoguchi E, et al. Role of tumor necrosis factor receptor 2 (TNFR2) in colonic epithelial hyperplasia and chronic intestinal inflammation in mice. Gastroenterology. 2002;122(1):134-144.

61. Fukata M, et al. Innate immune signaling by Tolllike receptor-4 (TLR4) shapes the inflammatory microenvironment in colitis-associated tumors. Inflamm Bowel Dis. 2009;15(7):997-1006.

62. Corredor J, et al. Tumor necrosis factor regulates intestinal epithelial cell migration by receptordependent mechanisms. Am J Physiol Cell Physiol. 2003;284(4):C953-961.

63. Perez-Moreno M, Song W, Pasolli HA, Williams SE, Fuchs E. Loss of p120 catenin and links to mitotic alterations, inflammation, and skin cancer. Proc Natl Acad Sci U S A. 2008;105(40):15399-15404.

64. Oshima $M$, et al. Suppression of intestinal polyposis in Apc delta716 knockout mice by inhibition of cyclooxygenase 2 (COX-2). Cell. 1996;87(5):803-809.

65. Hull MA, et al. Regulation of stromal cell cyclooxygenase- 2 in the ApcMin/+ mouse model of intestinal tumorigenesis. Carcinogenesis. 2006;27(3):382-391.

66. St-Onge $M$, et al. Characterization of prostaglandin E2 generation through the cyclooxygenase (COX)2 pathway in human neutrophils. Biochim Biophys Acta. 2007;1771(9):1235-1245.

67. Brown MV, Burnett PE, Denning MF, Reynolds AB. PDGF receptor activation induces $\mathrm{p} 120$-catenin phosphorylation at serine 879 via a PKCalpha-dependent pathway. Exp Cell Res. 2009;315(1):39-49.

68. Parkos CA, Delp C, Arnaout MA, Madara JL. Neutrophil migration across a cultured intestinal epithelium. Dependence on a CD11b/CD18-mediated event and enhanced efficiency in physiological direction. J Clin Invest. 1991;88(5):1605-1612. 